\title{
Deutsche Initiative für Netzwerkinformation (DINI): Lernen 4.0 - Wettbewerb um die besten Lernkonzepte
}

\author{
Die Deutsche Initiative für Netzwerkinformation (DINI) sucht \\ Ideen für neuartige Lernkonzepte an Hochschulen und \\ Universitäten.
}

http://doi.org/10.1515/bd-2019-0014

Augmented-Reality-Apps, 3D- und Videobrillen - digitale Werkzeuge verändern die Lehre an Hochschulen und Universitäten. Doch was halten die Studierenden von digitalen Lehr- und Lernmethoden? Die Deutsche Initiative für Netzwerkinformation (DINI) sucht in einem studentischen Wettbewerb nach den besten Ideen für das Lernen der Zukunft.

Unter dem Motto „Lernen 4.0 - Gestalte Deinen Lernraum“ werden

- Ideen für individuelle oder kooperative Lernumgebungen,

- Konzepte für Online-Lernräume oder physische Lernräume,

- Anwendungen von Virtual- oder Augmented Reality für die Lehre sowie

- innovative Lernkonzepte oder persönliche Lerntools gesucht.

DINI-Vorsitzender Dr. Helge Steenweg sagt zum Start des Wettbewerbs: „Wir freuen uns über ideenreiche Impulse der Studierenden zur Weiterentwicklung von digitalen und analogen Lernkonzepten. Als Dienstleister für die Hochschullehre arbeiten wissenschaftliche Bibliotheken, Medienzentren und Rechenzentren ständig an der Weiterentwicklung ihrer Angebote. Der DINI-Wettbewerb sammelt Vorschläge zur Verbesserung der Lehre, prämiert die besten Ideen und fördert den Transfer der studentischen Visionen in die Praxis der wissenschaftlichen Einrichtungen in Deutschland."

Bis zum 1. Juni 2019 können sich Studierende aller Hochschulen in Deutschland als Einzelpersonen oder Teams an dem Wettbewerb beteiligen. Die Visionen können als Ideenskizzen, Konzeptarbeiten oder andere kreative Beiträge in analoger und digitaler Form eingereicht werden. Kriterien des Wettbewerbs sind Innovation, Kreativität, Usability, Realisierbarkeit und Reichweite der eingereichten Beiträge. Eine Jury aus Expertinnen und Experten wird die eingereichten Beiträge begutachten. Die beste Idee wird mit 5.000 Euro prämiert. 
Die Mitglieder der Jury sind:

- Oliver Janoschka - Stifterverband, Geschäftsführer des Hochschulforums Digitalisierung,

- Franziska Kocks - Studentin der Universität Kiel und eine Gewinnerin des letzten Wettbewerbs,

- Prof. Dr. Kerstin Mayrberger - Universität Hamburg, Professorin für Lehren und Lernen an der Hochschule mit dem Schwerpunkt Mediendidaktik,

- Prof. Dr. Gerhard Peter - Mitglied des DINI-Beirats und Professor für Informatik (emeritiert),

- JProf. Dr. Mandy Schiefner-Rohs - Technische Universität Kaiserslautern, Juniorprofessorin für Pädagogik mit Schwerpunkt Schulentwicklung sowie

- Dr. Helge Steenweg - DINI-Vorsitzender und Direktor der Universitätsbibliothek Stuttgart.

Die Preisverleihung erfolgt im Rahmen der DINI-Jahrestagung 2019, die vom 8. bis 9. Oktober 2019 an der Universität Osnabrück stattfindet. Die Finalistinnen und Finalisten werden zu dieser Tagung eingeladen, um ihre Arbeiten dort Vertreterinnen und Vertretern von wissenschaftliche Bibliotheken, Medienzentren und Rechenzentren vorzustellen.

Der DINI-Wettbewerb sammelt zum fünften Mal Ideen zur Verbesserung des digital unterstützten Lehrens und Lernens, prämiert die besten Ideen und fördert den Transfer der studentischen Visionen in die Praxis der wissenschaftlichen Einrichtungen in Deutschland.

Weitere Informationen finden sich unter: https://www.dini.de/wettbewerbe/ lernen-40-gestalte-deinen-lernraum/. 